\title{
DWT for Image and Video Compression using MATLAB
}

\author{
P. Sreekanth \\ CVR College of Engineering, ECE Department, Hyderabad, India \\ Email: sreekanth.isoft@gmail.com
}

\begin{abstract}
Amount of data required to represent an image can be reduced by removing repetitive information which is known as compression of images. Today with the increase in the usage of computer, compression is very necessary. Because the space required for holding the uncompressed image outlays more. Fortunately, today image compression can be achieved by various methods. Although Video looks like ceaseless gesture, it is actually a series of halcyon images. The paper describes about the application of DWT methods for image and video compression. We also computed the PSNR and CR values after image reconstruction using IDWT algorithm.
\end{abstract}

Index Terms-power, energy, Image compression, video compression, Discrete Wavelet Transform (DWT), Inverse Discrete Wavelet Transform (IDWT), Peak signal Noise Ratio (PSNR) and Compression Ratio (CR)..

\section{INTRODUCTION}

One image equals more than thousand words, unfortunately storing image outlays more than million words. Compression of image makes faster loading of web pages and it also saves a lot of bandwidth. Compression of image makes people to send photos easily which in turn reduces the bandwidth outlays and not make the recipient of the email angry. Therefore, compression is a necessary and essential for creating image files with feasible and transmittable sizes.

Video is a series of halcyon images which are called frames [5]. The consumer's use of the digital video increasing day by day, so video compression is necessary to curtail the size. There are two imperative benefits provided by video compression. First, it makes it potential to use the digital video transmission and storage environments that would not support uncompressed video. For example, modern Internet throughput rates are insufficient to handle uncompressed video in real time. Storage of uncompressed video on a DVD is possible only for a few seconds, so storage of video can be practical with video and audio compression. Secondly efficient usage of transmission and resources can be achieved by compression of video. If a high bit tare transmission channel is available, then it is a more alluring proportion to send a high resolution If a high bit rate transmission channel is available, then it is a more alluring proposition to send a high resolution compressed $\mathrm{v}$ ideo or multiple compressed video channels than send a single, low resolution, uncompressed stream. Even with consistent advances in the stockpile and communication capacity, the essential component of multimedia services for many years to come is compression. In a listless compression system analytical repetition is removed so that the authentic signal can be perfectly reconstructed at the receiver. Unfortunately, at the present time lossless methods can achieve only small amount of compression of image and video signals. Most practical video compression techniques are based on the lossy compression, in which preeminent compression is achieved, but decoded signal not identical to the authentic. A video compression algorithm is used for adequate compression with less low.

\section{ONE-DIMENSIONAL DWT AND IDWT ARCHITECTURE}

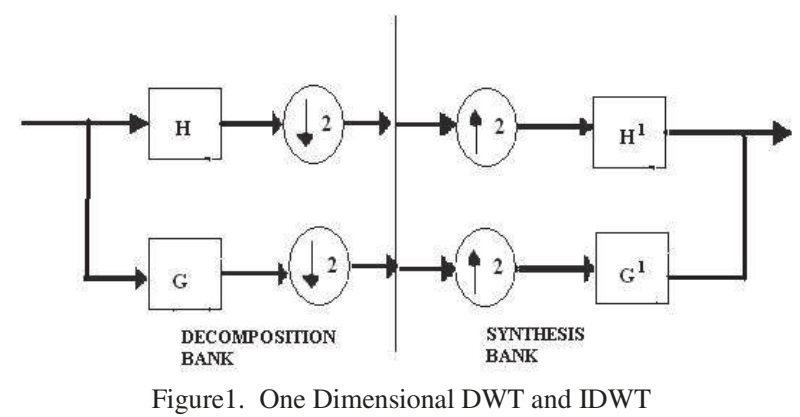

The universal form of 1 D DWT is shown in above figure [3]. Here the discrete signal is passed through a low pass and high pass filters $\mathrm{H}$ and $\mathrm{G}$ then down sampled by factor 2 completes forward wavelet transform. The inverse is obtained by up sampling by a factor of 2 and then using the reconstruction filters $\mathrm{H} 1$ and $\mathrm{G} 1$. The low pass $(\mathrm{H})$ and high pass filter $(\mathrm{G})$ combined called as decomposition (analyze) filter bank [6]. The low pass (H1) and high pass (G1) filters are combined called as the Synthesis filter bank [6]. Analyze filter banks are used for compression and synthesis filter bank is used for decompression

\section{TWO-DIMENSIONAL DWT AND IDWT ARCHITECTURE}

Image is two dimensional signal which is denoted by $\mathrm{X}(\mathrm{m}, \mathrm{n})$ here $\mathrm{m}$ is the number of rows and $\mathrm{n}$ is the number of columns. So for image compression, first we apply DWT Algorithm to rows followed by columns. We can interchange the order of rows and columns means we first apply DWT algorithms to the column first followed by rows next. Similarly, IDWT algorithm also applied to the 
columns followed by rows completes the reconstruction of images. The following figure shows the level one DWT and IDWT architecture for images [3], [4].

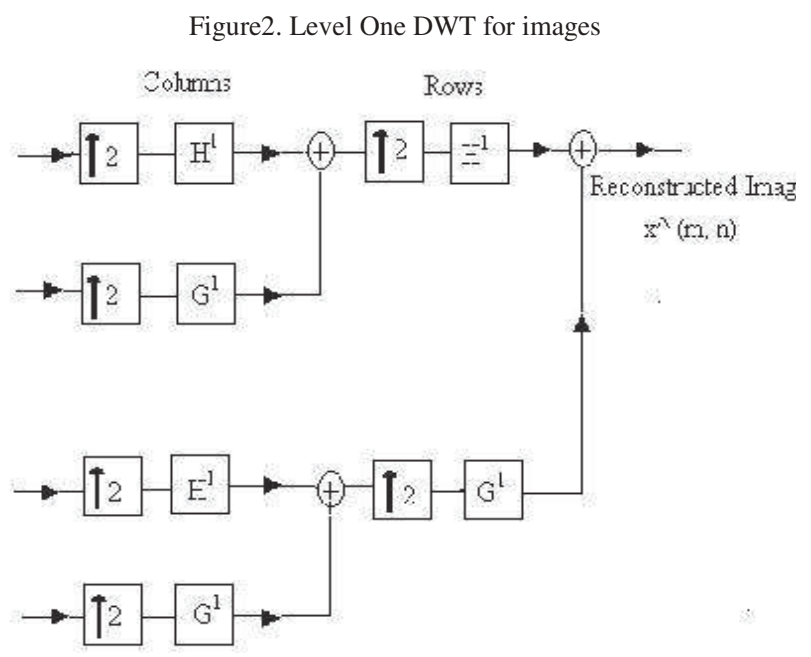

Figure3. Level One IDWT for images

High pass filter output values are called as "detail coefficients" [3] and the output values of low pass filter called as approximate coefficients [3]. Detail coefficient values are less significant in image reconstruction even though we neglect these values we get back the original image with less loss.

\section{DWT FOR 2 D IMAGES}

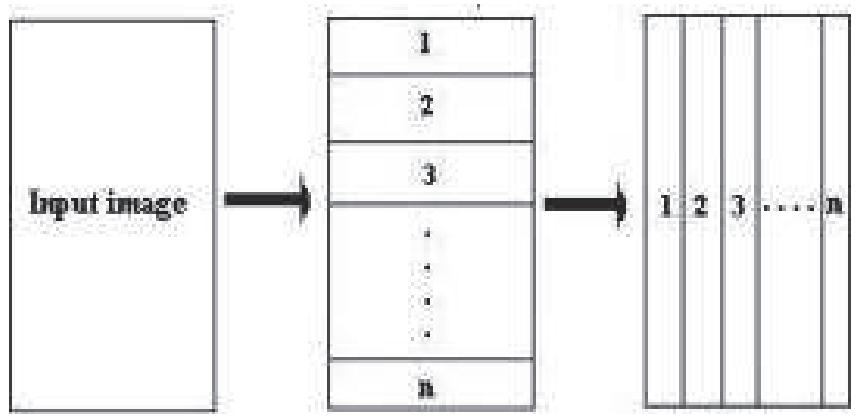

Figure4. Applying DWT to images for compression

Wavelet transformation entails transformation of image data horizontally first and then vertically. Here the image plane is divided into $\mathrm{n}$ horizontal sections which are horizontally transformed concurrently. After then the image is divided into $\mathrm{n}$ vertical sections which are then vertically transformed concurrently. It is not a must that the number of horizontal sections is equal to the number of vertical sections.

\section{CR AND PSNR}

A touchstone in compression of image data is the compression ratio and PSNR (Peak Signal to Noise Ratio) [5]. The compression ratio is used to measure the amount of data compressed by comparing the size of the compressed image and original image. The preeminent the compression ratio means the better the wavelet functions.
PSNR is another important parameter used to calibrate the image nature [8]. PSNR parameter is always used as a touchstone to find the closeness between reconstructed and the original image. Better image quality can be achieved by larger PSNR value [1].

$$
\begin{aligned}
& \text { Compression Ratio }=\frac{\text { The size of compressed Image }}{\text { The size of the Original Image }} \\
& \text { PSNR defined as follows [2], [3] } \\
& P S N R=10 \cdot \log \left|\frac{255}{\sqrt{\mathrm{MSE}}}\right|
\end{aligned}
$$

Where MSE is mean square error,

$$
\left.\mathrm{MSE}=\sum_{\mathrm{m}=1}^{\mathrm{M}} \sum_{\mathrm{n}=1}^{\mathrm{N}}\left(\mathrm{X}(\mathrm{m}, \mathrm{n})-\mathrm{X}^{\wedge}(\mathrm{m}, \mathrm{n})\right)\right)^{2}
$$

\section{IMAGE COMPRESSION RESULTS}

\section{A) Level - One Compression results}

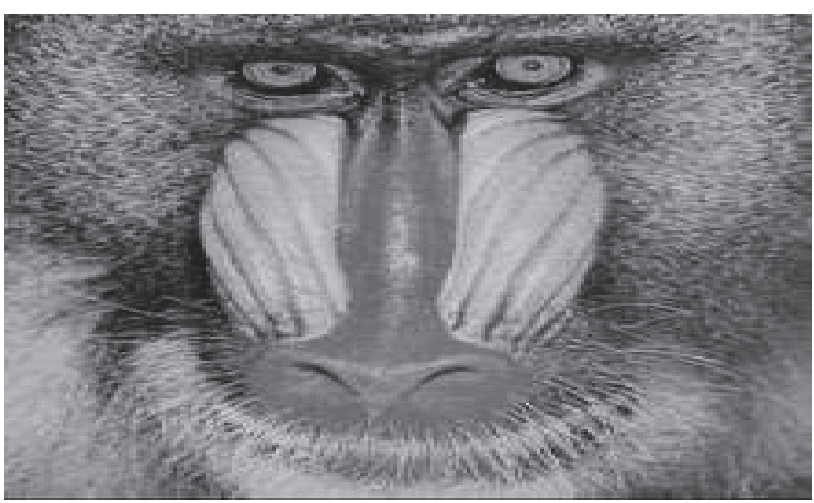

Figure5 (a) baboon image

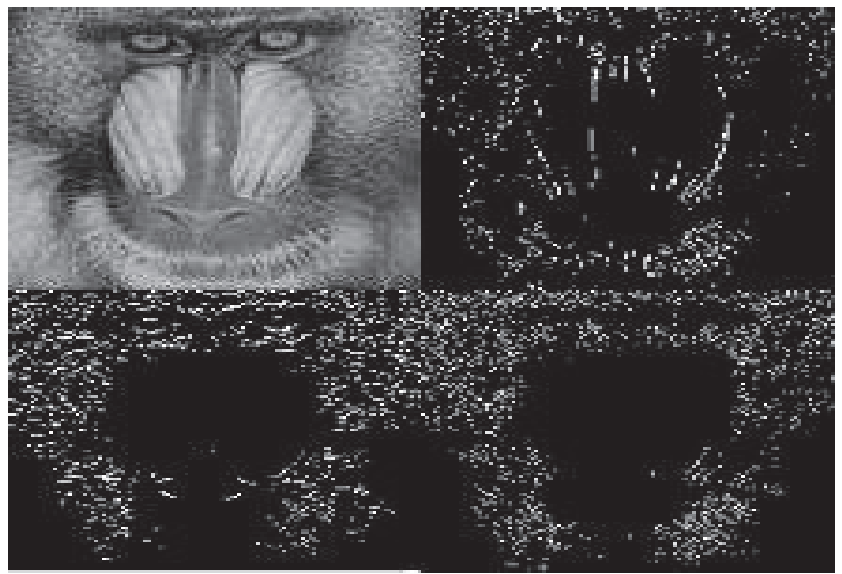

Figure4 (b) Level one DWT

Figure5 (b) Level one DWT

(Detail coefficient values enhanced by thresholding) 


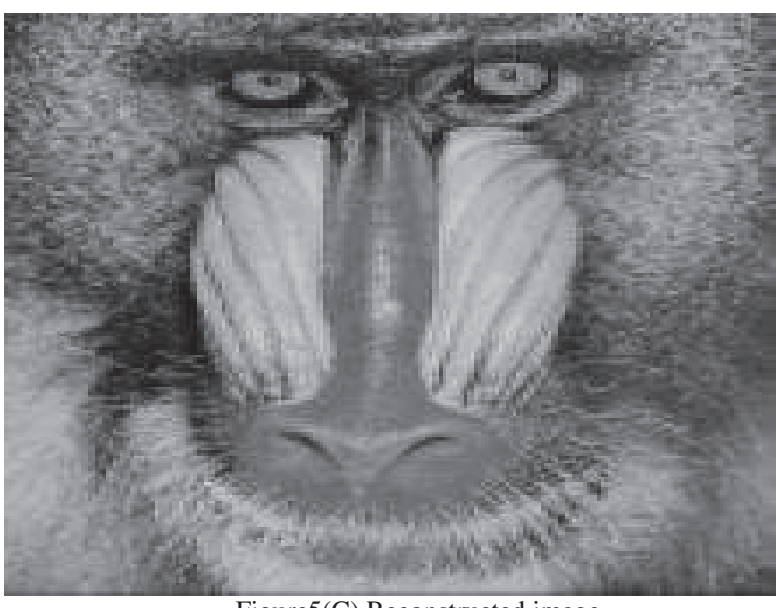

Figure5(C) Reconstructed image

(Only Approximate coefficient values are used)

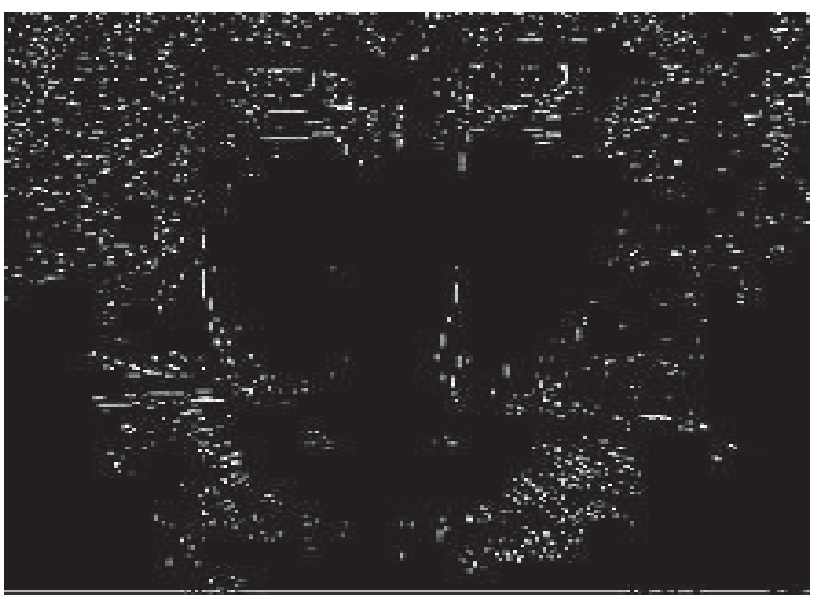

Figure5 (d) Difference between original and reconstructed (Coefficient values enhanced by thresholding)

Here we got the compression Ratio of ' 4 ' and PSNR $34.1 \mathrm{~dB}$. Here the detail coefficients values are enhanced for better view. Thresholding mechanism it used to enhance the values. Here threshold value is 20 , means all the above 20 are forced to 255 and below 20 are forced to zero.

\section{B) Level - Two Compression Results}

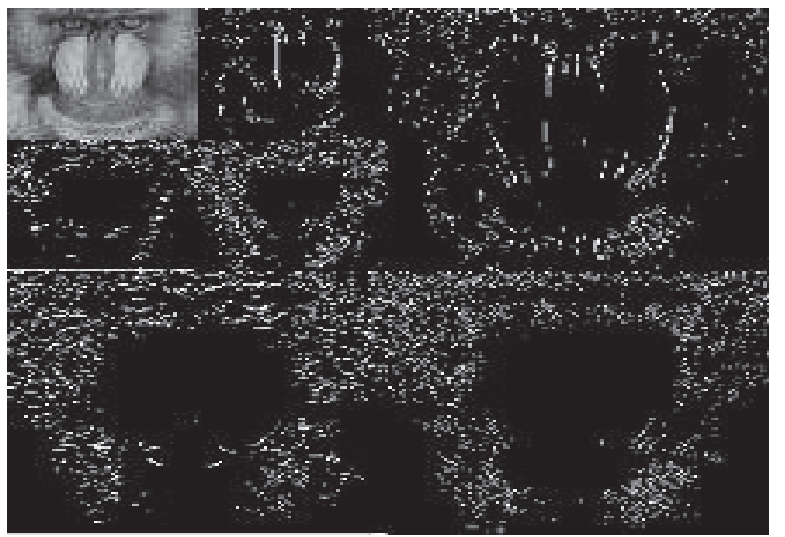

(Detail coefficient values enhanced by thresholding)

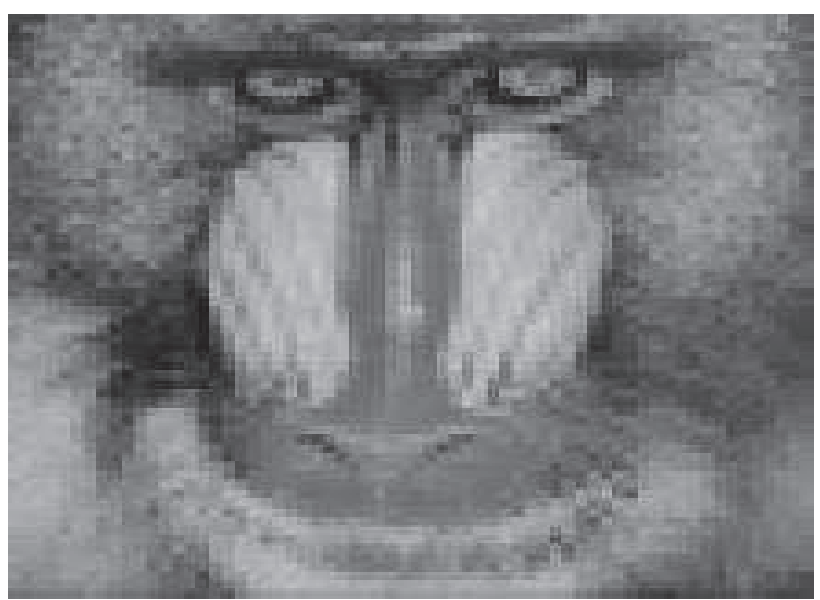

Figure6 (b) Reconstructed images

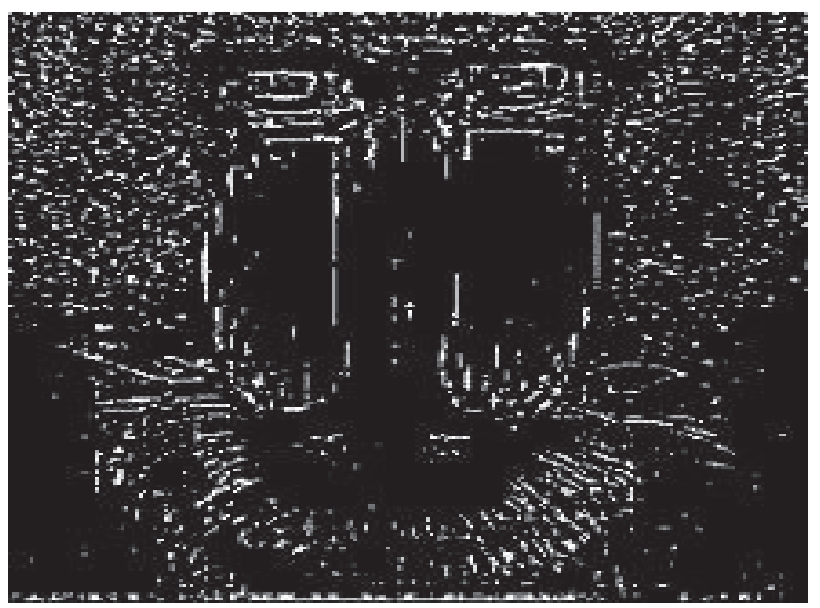

Figure6 (C) Difference between original and reconstructed (Coefficient values enhanced by thresholding)

Here we got the compression Ratio of ' 16 ' and PSNR of $32.44 \mathrm{~dB}$. Here the detail coefficients values are enhanced for better view with the threshold value of 20.The difference between authentic and reconstructed image figure (5(c)) increased in level two DWT results compared to level one DWT results (figure(5(d)). Observe that the PSNR value is decreased in level two DWT means the reconstructed image quality is less. As compression Ratio increases, the reconstructed image quality decreases.

\section{VIDEO COMPRESSION RESULTS}

A) Level - one Video Compression Results

Although video look like ceaseless gesture, it is actually a series of halcyon images, and changing fast enough that it look like ceaseless motion, so video compression is similar to image compression. Video compression is done when we compress all the frames in that video. Here I have taken VIP TRAFFIC video which consists of 120 frames in it. Here some of frames results are shown.

Figure6 (a) Level Two DWT image 

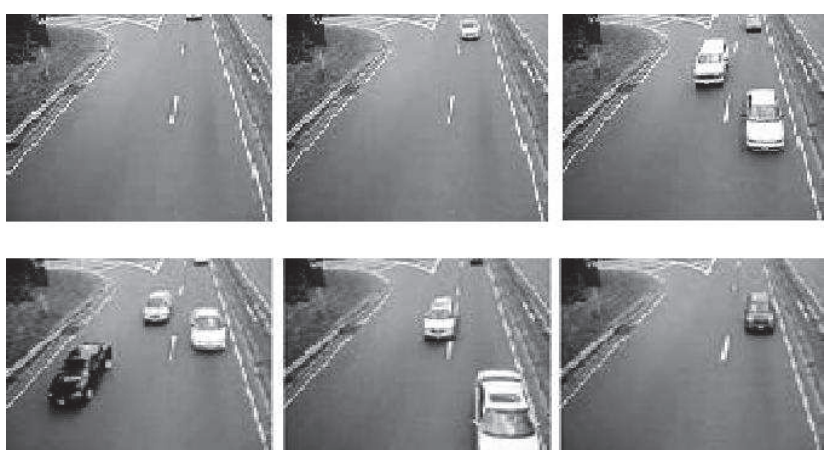

Figure6 (a) some frames in Video Traffic
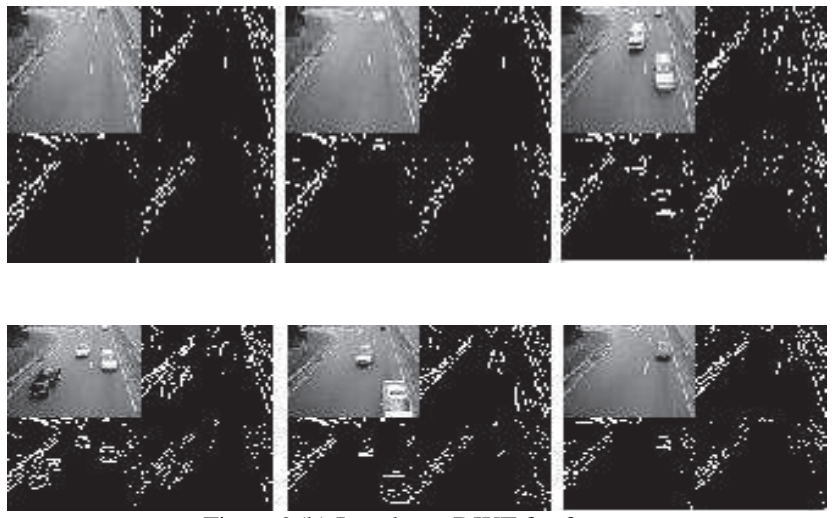

Figure6 (b).Level one DWT for frames

(Detail coefficient values enhanced by thresholding)

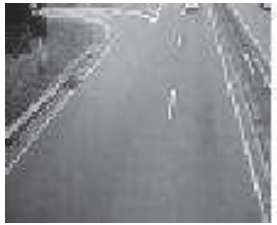

$P S N R=39.83 \mathrm{~dB}$

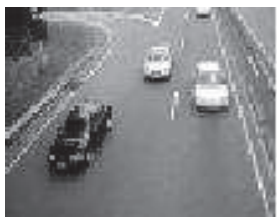

PSNR $=38.35 \mathrm{~dB}$

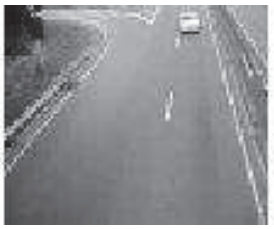

PSNR=39.83 dB

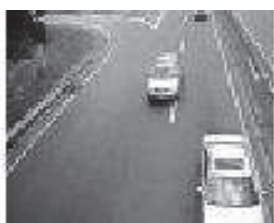

PSNR=39.83 dB

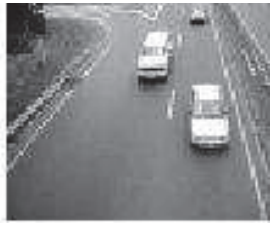

$P S N R=38.02 \mathrm{~dB}$

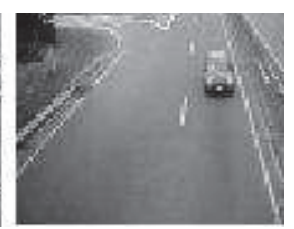

PSNR= $36.74 \mathrm{~dB}$

Figure6 (c). Reconstructed frames with PSNR values

(Only approximate coefficients are used)

B) Level - Two Video Compression Results
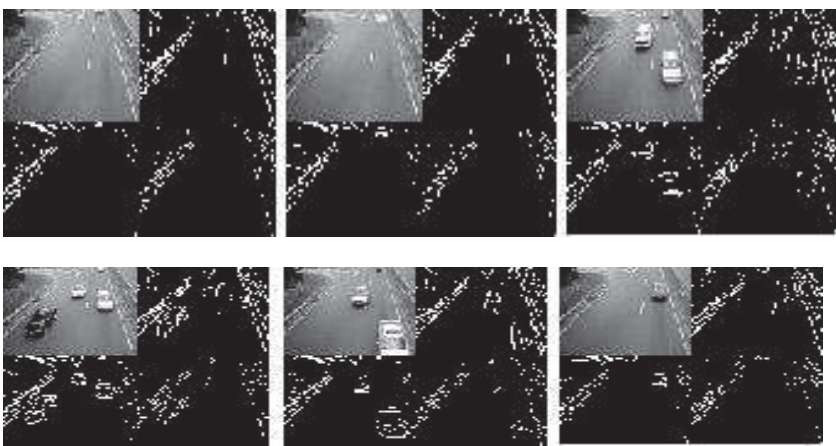

7 (a).Level -Two DWT for video frames

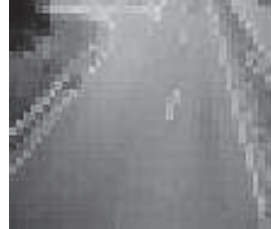

PSNR=32.84 dB

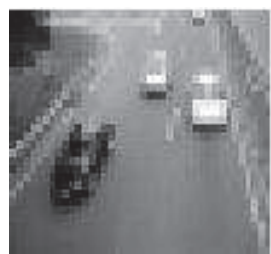

PSNR $=33.29 \mathrm{~dB}$

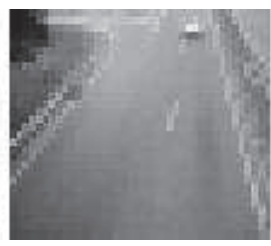

PSNR=32.84 dB

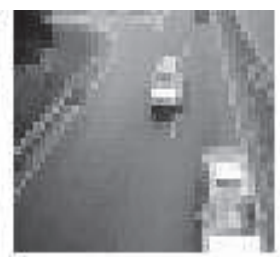

PSNR=34.25 dB

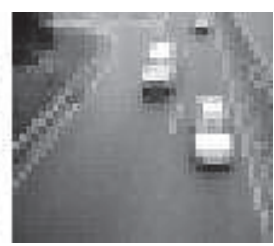

PSNR=33.10 dB

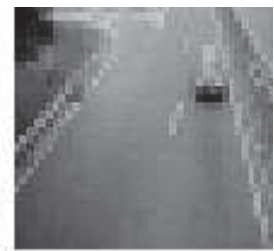

PSNR=34.45 dB
Figure7 (b). Reconstructed frames with PSNR values (Only approximation coefficients are used)

Compression Ratio (CR) is 4 for one level compression and 16 for two level compressions. PSNR value decreasing in level two compressions compared to level one compression, it means the reconstructed image quality decreasing and error between original and reconstructed image is increasing. Larger PSNR will produce better image quality

TABLE 1

Results of video Frames

\begin{tabular}{|l|l|l|}
\hline $\begin{array}{c}\text { Compression } \\
\text { Ratio }\end{array}$ & \multicolumn{1}{|c|}{ PSNR(DB) } & $\begin{array}{c}\text { Level Of } \\
\text { DWT }\end{array}$ \\
\hline 4 & 39.83 & 1 \\
\hline 16 & 33.83 & 2 \\
\hline
\end{tabular}

\section{CONCLUSIONS}

Digital video compression techniques have played an important role in the world of telecommunication and multimedia systems where bandwidth is still a valuable commodity. Hence, video compression techniques are of prime importance of reducing the amount of information needed for picture sequence without losing much of its quality, judged by human viewers. Here we have used Discrete Wavelet Transform (DWT) to achieve the compression for the image and it is extended to a series of images which is nothing but a video. PSNR and Compression Ratio are calculated in this paper. PSNR value is decreasing as the compression ratio is increasing, this means that the reconstructed image quality is decreasing.

\section{REFERENCES}

[1] Ratchakit Sakuldee and Somkait Udomhunsakul, "Objective Performance of Compressed Image QualityAssessments”,World Academy of Science, Engineering and Technology 352007.

[2] http://en.wikipedia.org/wiki/Discrete_wavelet_transform. 
[3] Jyotishman Das, "Lossless performance of image compression using 2D DWT.

[4] Iain E. G. Richardson, "H.264 and MPEG

Video Compression", Wiley Publication,2003.

[5] Sanjay kumar Mitra, "Digital Signal Processing", Fourth edition, pp 15- 56,2010. 